\title{
Challenges of ELR Implementation: Moving Toward Semantic Understanding through Vocabulary Validation
}

\author{
Daniel B. Golson*, Natalie Raketich and Erin Holt \\ Communicable and Environmental Diseases and Emergency Preparedness, Tennessee Department of Health, Nashville, TN, USA
}

\section{Objective}

To develop a means for validating standardized vocabulary used to report laboratory events via prescribed electronic laboratory reporting (ELR) standards and implementation guides in order to limit internally inconsistent information within ELR messages intended for public health action.

\section{Introduction}

Meaningful Use has increased interest in submission of ELR to public health agencies, prompting these agencies to analyze their reporting process. Tennessee's reporting regulations require anyone with knowledge of or suspecting a reportable disease or event report to the local health department. Although it is understood that laboratories are more diligent and routine reporters, focus in listing of these events is from the healthcare provider perspective. Public health agencies must acknowledge the differences in provider case reporting and laboratory result reporting.

Despite Tennessee Department of Health's (TDH) required use of standardized vocabulary for ELR such as Logical Observation Identifiers Names and Codes (LOINC) to identify the test performed and Systematized Nomenclature of Medicine-Clinical Terms (SNOMED-CT) to identify organism names, ordinal results, and specimen type, internally inconsistent information in messages has been identified. For example, a performed test with LOINC value 13950-1 encodes for a hepatitis A virus IgM antibody test from serum or plasma using an enzyme immunoassay (EIA) and calls for an ordinal result. However the sender describes a "Hepatitis C Antibody (Anti HCV)" test and provides a numeric result. In order to achieve semantic understanding of the actionable content of ELR messages, a systematic means to document and validate vocabulary is needed.

\section{Methods}

To develop a vocabulary validation process, we determined who within TDH required input in determining laboratory observations of interest for public health investigation and disease surveillance as some reportable events cross multiple program areas. These subject matter experts consulted published case definitions and identified their business needs.

From these information-gathering sessions, a laboratory guidance document was developed, organized from the laboratory observation point of view which cross-linked to the public health case observation providers were used to seeing from TDH. Included in the document are specific details about laboratory observations of interest and constraints upon them such as specimen source. This document was then used to develop a collection and validation tool for on-boarding trading partners looking to send ELR for reportable events to TDH.

\section{Results}

TDH has developed an ELR on-boarding process which places vocabulary validation at the beginning. Potential ELR submitters are asked to complete a vocabulary validation worksheet which captures information related to reportable laboratory observations based on data elements specified in ELR Health Level Seven (HL7) messages including test performed, observation made, and specimen type. This process allows TDH to better understand the mapping of local codes to standardized codes and helps identify potential internally inconsistent information in ELR messages before entry into production environments of disease surveillance systems. TDH works with laboratories to complete the worksheet and agree upon vocabulary, recognizing that the process is iterative and can be time-consuming for both parties, but is critical to effective understanding and processing of the content of ELR.

\section{Conclusions}

Working with ELR reporters to populate the vocabulary validation worksheet and resolve internal discrepancies allows TDH to better understand the content of ELR received before it is used by public health staff for public health surveillance, investigation, and intervention purposes. Receipt of internally inconsistent information in ELR messages causes confusion as to which piece of data is "more correct" which affects the ability of public health to understand and process information received. Use of standardized codes is only useful when the codes convey the information they are intended to convey. When descriptions of tests and standardized codes they are mapped to contradict each other or other information contained in the ELR message such as result or specimen, ELR has the potential to drain limited time and resources available to public health to conduct its mission. In order to hasten on-boarding and realize the benefits of ELR to public health practice, it is imperative to document and validate the actionable content of ELR messages early in the process.

\section{Keywords}

Electronic Laboratory Reporting; Standards; Public Health Surveillance; Vocabulary; Data Quality

\author{
*Daniel B. Golson \\ E-mail: daniel.golson@tn.gov
}

